\title{
«C'est la démocratie»
}

\section{Interview: Bruno Kesseli}

(Dr med. et lic. phil., rédacteur en chef

Lors de la prochaine session d'hiver, le Conseil national doit statuer sur la loi sur les produits du tabac. Contrairement au Conseil des Etats, la majorité de la Commission de la santé publique du Conseil national (CSSS-N) présidée par le conseiller national Ignazio Cassis ne souhaite pas que la loi soit renvoyée au Conseil fédéral. Dans une brève interview, l'ancien médecin cantonal tessinois explique ce que ce choix signifie pour la loi.

Ignazio Cassis, contrairement au Conseil des Etats, la Commission de la santé publique du Conseil national (CSSS-N) ne veut pas renvoyer la loi sur les produits du tabac au Conseil fédéral pour qu'elle soit remaniée. Est-ce que cela augmente les chances que le projet du Conseil fédéral soit accepté?

Cela permettrait surtout de ne pas perdre de temps. Un renvoi retarderait le projet d'environ deux ans. En ce qui concerne les produits du tabac, nous devons combler une lacune juridique. En effet, ils étaient jusqu'à présent régis par la loi sur les denrées alimentaires, dont ils ont été retirés lorsqu'elle a été révisée. Si le Parlement estime que la proposition de loi sur les produits du tabac du Conseil fédéral va trop loin, il peut procéder lui-même aux amendements nécessaires. Un renvoi signifie au contraire: «Il faut intervenir, mais pas de cette manière. Le Conseil fédéral doit lui-même mettre au point une nouvelle proposition, en tenant compte des directives du Parlement.» Mais d'un point de vue technique, cette loi est assez simple. Le Parlement peut très bien prendre les décisions requises. Par ailleurs, la population pourra avoir le dernier mot à travers un référendum.

Le Conseil des Etats s'est exprimé contre une interdiction de la publicité vantant les produits du tabac, argumentant que l'économie de libre-marché doit prévaloir sur la prévention. Une telle affirmation n'est-elle pas cynique lorsqu'elle se rapporte à des produits dont l'impact négatif sur la santé est prouvé?

Si, dans un Etat libéral, on interdisait tout ce qui est nocif pour la santé, il n'y aurait plus d'Etat libéral. La liberté - y compris celle de se faire du mal - est en effet jugée plus importante que la santé dans de nombreuses parties du monde. Beaucoup de peuples se sont battus pour cela pendant des siècles. La préven-

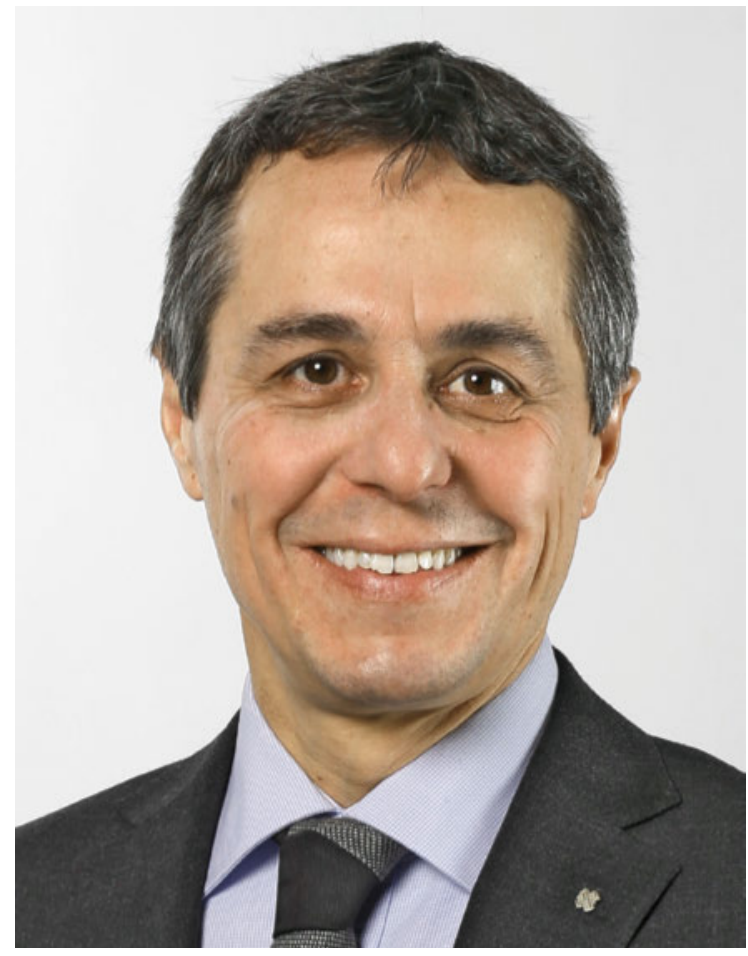

"Un référendum est probable»: Ignazio Cassis, conseiller national.

tion est aujourd'hui une notion positive. Mais l'une de ses dérives peut être la mise sous tutelle des gens, comme on l'a vu par le passé. C'est encore le cas aujourd'hui en ce qui concerne la politique en matière de drogues. L'Etat doit-il protéger chaque individu contre lui-même? De quelle part de mise en danger de soi et de responsabilité personnelle une société veut-elle disposer? Où se situe la limite? Ce sont des questions qui agitent le Parlement et auxquelles les différents partis politiques présentent des réponses hétérogènes. C'est la démocratie. 
Etant donné les rapports de force actuels au Parlement, une loi sur les produits du tabac "efficace" en termes de prévention et de protection complète de la jeunesse, interdiction de la publicité comprise, est-elle seulement envisageable?

Nous devons d'abord voir si le Conseil des Etats maintient sa décision de renvoi. Si tel est le cas, le projet sera renvoyé au Conseil fédéral assorti de certaines conditions. En feront partie la protection de la jeunesse avec l'ancrage national de l'âge minimal de 18 ans pour l'achat de produits du tabac, la création de bases légales pour les achats tests et l'interdiction de la publicité qui s'adresse spécialement aux mineurs. Le Conseil des Etats ne souhaite toutefois pas de restrictions supplémentaires de la publicité, de la promotion des ventes et du parrainage. Il estime qu'une interdiction générale de la publicité irait trop loin dans le cas des adultes. Une importance prépondérante a ici été accordée à la liberté.

\section{La politique est l'art du réalisable. En ce qui concerne la loi sur les produits du tabac, qu'est-ce qui est selon vous réalisable en Suisse?}

Si le Conseil national confirme la décision de sa Commission de ne pas renvoyer la loi en décembre, le Conseil des Etats devra l'examiner à nouveau. Si le projet actuel est discuté, je m'attends à ce que les restrictions en termes de publicité, de promotion des ventes et de parrainage des produits du tabac soient assouplies et à ce que l'approche fédéraliste soit renforcée. Il n'est pas sûr qu'à la fin, la loi soit toujours compatible avec la FCTC**. Et la décision finale reviendra au peuple: vu le sujet délicat, un référendum est probable. Mais chaque chose en son temps, il s'agit de conserver son optimisme!

\section{Crédit photo}

Photo mise à disposition par Ignazio Cassis

\section{Convention-cadre de I'OMS pour la lutte} antitabac

(Source: Office fédéral de la santé publique OFSP; http://www. bag.admin.ch/themen/drogen/00041/04133/14737/index.

html?lang=fr)

La Convention-cadre de I'OMS pour la lutte antitabac (CCLAT, en anglais: Framework Convention on Tobacco control, FCTC) est le premier traité international négocié sous les auspices de I'OMS (premier traité mondial de santé publique). Elle énonce les principes qui s'appliquent, au niveau international, à la vente et à la consommation de produits du tabac. Les différentes mesures, basées sur des connaissances scientifiques, visent à réglementer l'offre et la demande des produits du tabac, dans l'objectif d'améliorer l'état de santé de la population mondiale.

La CCLAT a été adoptée par l'Assemblée mondiale de la Santé le 21 mai 2003 et est entrée en vigueur le 27 février 2005. Elle est devenue depuis l'un des traités ayant remporté la plus rapide et la plus large adhésion dans I'histoire des Nations Unies.

Elle a été signée par 168 Etats, puis ratifiée par 180 pays y compris par I'Union européenne (état: mars 2016).

La CCLAT a été élaborée suite à la forte augmentation, au niveau mondial, des maladies et décès liés à la consommation de tabac. Elle réaffirme le droit de tous les peuples au niveau de santé le plus élevé possible. La CCLAT représente un jalon dans la promotion de la santé publique et apporte une dimension juridique nouvelle à la coopération internationale en matière de santé.

La CCLAT contraint les Parties à prendre notamment les mesures suivantes:

Ordonner des mesures de protection efficaces contre le tabagisme passif sur les lieux de travail, dans les espaces clos, les transports en commun et les lieux publics.

Veiller à l'étiquetage et faire figurer des mises en garde sur l'ensemble des produits du tabac.

Restreindre la publicité et le parrainage en faveur du tabac. Lutter contre la contrebande, la fabrication illicite et la contrefaçon des produits du tabac.

Interdire la vente de produits du tabac aux mineurs.

La Suisse a signé la Convention le 25 juin 2004, exprimant ainsi la volonté du Conseil fédéral de mettre en œuvre le projet de I'OMS en Suisse. L'objectif à terme du Conseil fédéral est la ratification.

WHO Framework Convention on Tobacco Control: http://apps. who.int/iris/bitstream/10665/42811/1/9241591013.pdf?ua=1 